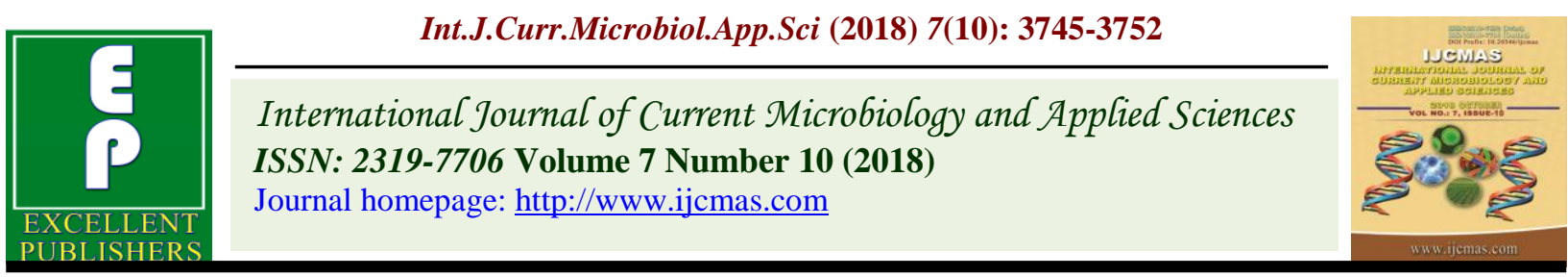

\title{
Weed Management Practices on Nutrient Uptake, Growth Parameters and Yield in Kharif Season Brinjal
}

\author{
K. Vidyasagar, R. V. S. K. Reddy, K. Venkata Subbaiah*, \\ M. Madhavi and S. S. Vijayapadma
}

D. Y.S.R. Horticultural University, Venkataramannagudem, India

*Corresponding author

\begin{tabular}{|c|}
\hline Keywords \\
\hline $\begin{array}{l}\text { Brinjal, Weed } \\
\text { management, Growth } \\
\text { parameters, Nutrient } \\
\text { uptake, Yield }\end{array}$ \\
\hline Article Info \\
\hline $\begin{array}{l}\text { Accepted: } \\
\text { 20 September } 2018 \\
\text { Available Online: } \\
10 \text { October } 2018\end{array}$ \\
\hline
\end{tabular}

\section{Introduction}

Brinjal is native to India. It belongs to the family Solanaceae and it is also called as eggplant or aubergine. In India it is grown in an area of 589.5 thousand hectares, producing $10,163.1$ thousand tonnes with a productivity of $17,240 \mathrm{~kg} \mathrm{ha}^{-1}$. In Andhra Pradesh it is grown in an area of 24.30 thousand hectares, producing 485.50 thousand tonnes with a productivity of $19,959 \mathrm{~kg} \mathrm{ha}^{-1}$ (CMIE-2010).

\begin{abstract}
An experiment was conducted on weed management practices on weed control efficiency, growth parameters and yield in kharif season brinjal at Vegetable Research Station, Agriculture Research Institute, Rajendranagar, Hyderabad, during kharif. The experiment was laid out in randomized block design with thirteen treatment combinations and replicated thrice. Among the treatments, significantly the highest nitrogen uptake (180.45 $\left.\mathrm{kg} \mathrm{ha}^{-1}\right)$, phosphorus uptake $\left(142.22 \mathrm{~kg} \mathrm{ha}^{-1}\right)$, potassium uptake $\left(95.85 \mathrm{~kg} \mathrm{ha}^{-1}\right)$ was observed with hand weeding three times at 20, 40 and 60 days after transplanting. With respect to growth parameters, treatment $\mathrm{T}_{6}$ recorded the highest plant height at 20 Days after transplanting (DAT) $\left(17.38 \mathrm{~cm}\right.$ ) and Treatment $T_{12}$ (hand weeding three times at 20, 40 and 60 DAT) had highest plant height $(31.85 \mathrm{~cm}$.) at 40 Days after transplanting. The highest plant height at 60days after transplanting was recorded in pendimethalin C.S as pre-emergence @ $0.70 \mathrm{~kg} \mathrm{a.i} \mathrm{ha}{ }^{-1}+$ one hand weeding at $45 \mathrm{DAT}\left(\mathrm{T}_{1}\right)(56.52 \mathrm{~cm}$.). whereas at 80 days after transplanting, the highest plant height was found with hand weeding three times at 20,40 and 60 DAT (T12) $(74.69 \mathrm{~cm})$. Highest number of primary branches and plant spread were recorded in hand weeding three times at 20,40 and 60 DAT $\left(\mathrm{T}_{12}\right)(6.36),\left(0.34 \mathrm{~m}^{-2}\right)$. With respect to plant population, there was no significant difference between the treatments. The highest fruit yield $\left(31.10 \mathrm{t} \mathrm{ha}^{-1}\right)$ was noticed with hand weeding three times at 20,40 and 60 days after transplanting.
\end{abstract}


annual loss in brinjal yield due to weed menace. Hand weeding, though effective is laborious, cumbersome and under many situations it becomes uneconomical. Herbicides have become an important tool for weed control, but are not effective in controlling all the weeds present in the crop. Indeed very little work was done on weed management in brinjal. Hence the present investigation was undertaken to study the effect of weed management practices on weed control efficiency, growth parameters and yield in kharif season brinjal.

\section{Materials and Methods}

The field experiment was conducted at Vegetable Research Station, Agriculture Research Institute, Rajendranagar, Hyderabad, during kharif. The experimental site comes under sub-tropical zone and is situated at a latitude of $17^{0} 19^{1} \mathrm{~N}$ and longitude of $79^{0} 23^{1}$ E. The altitude of the place is $546.2 \mathrm{~m}$ above mean sea level. The mean annual precipitation on the basis of last ten years is $852 \mathrm{~mm}$ which received almost from South-West Monsoon during June to October. The mean annual minimum and maximum temperatures are $20.2^{\circ} \mathrm{C}$ and $32.6^{\circ} \mathrm{C}$ respectively. The humidity ranged from 44.5 per cent in summer and 79.4 per cent in rainy season. The experimental site thus has hot dry summer and moderate cold winter.

Thirty days old seedlings were transplanted in the main field at a spacing of $60 \mathrm{~cm} \times 50 \mathrm{~cm}$. Different herbicides with thirteen treatment combinations viz., $\mathrm{T}_{1}$ - Pendimethalin C.S as pre-emergence @ $0.70 \mathrm{~kg}$ a.i ha ${ }^{-1}+$ one hand weeding at 45 DAT, $\mathrm{T}_{2^{-}}$Oxadiargyl as preemergence @90 g a.i ha ${ }^{-1}+$ one hand weeding at $45 \mathrm{DAT}, \mathrm{T}_{3}$ - Oxyfluorfen as pre-emergence @ $0.15 \mathrm{~kg} \mathrm{a} .1 \mathrm{ha}^{-1}+$ one hand weeding at 45 DAT, $\mathrm{T}_{4}$-Pendimethalin C.S as pre-emergence @ $0.70 \mathrm{~kg}$ a.i ha ${ }^{-1}$ followed by quizalofop ethyl @50 g a.i ha ${ }^{-1}$ at 15 to $20 \mathrm{DAT}, \mathrm{T}_{5^{-}}$
Oxadiargyl as pre-emergence @ $90 \mathrm{~g}$ a.i ha ${ }^{-1}$ followed by quizalofop ethyl @ $50 \mathrm{~g} \mathrm{a} . \mathrm{hha}^{-1}$ at 15 to 20 DAT, T6-Oxyfluorfen as preemergence@0.15 kg a.i ha ${ }^{-1}$ followed by quizalofop ethyl @ $50 \mathrm{~g} \mathrm{a.i} \mathrm{ha}{ }^{-1}$ at 15 to 20 DAT, $\mathrm{T}_{7^{-}}$Pendimethalin C.S as preemergence@0.70 kg a.i ha ${ }^{-1}$ followed by propaquiza fop@62.5 g a.i ha ${ }^{-1}$ at 15 to 20 DAT, T8-Oxadiargyl as pre-emergence @ $90 \mathrm{~g}$ a.i ha ${ }^{-1}$ followed by propaquiza fop @ $62.5 \mathrm{~g}$

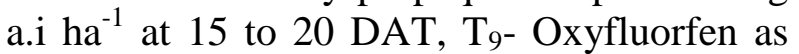
pre emergence@0.15 kg a.i ha ${ }^{-1}$ followed by propaquiza fop@62.5 g a.i ha ${ }^{-1}$ at 15 to 20 DAT, $\mathrm{T}_{10^{-}}$Glyphosate @ $1.5 \mathrm{~kg}$ a.i ha ${ }^{1}$ directed spray at 25 and $50 \mathrm{DAT}, \mathrm{T}_{11^{-}}$Inter cultivation at 25 and 50 DAT, $\mathrm{T}_{12}$-Hand weeding three times at 20,40 and 60 DAT and $\mathrm{T}_{13}$ - Control were tried and replicated thrice.

The required quantity of herbicides for the experimentation was calculated by using the following formula.

Commercial herbicide $=\frac{\text { RA }}{\text { C }}$

Here,

$\mathrm{R}=$ Recommended dose of herbicide per hectare

$\mathrm{A}=$ Area (hectares)

$\mathrm{C}=$ Concentration of herbicide

Thus calculated quantity of herbicide was sprayed in each treatmental plot using knapsack sprayer with flat pan nozzle a with spray volume of 600 litres per hectare. The quantity of herbicide per plot $(4.2 \mathrm{~m} \mathrm{x} 4 \mathrm{~m})$ was calculated by using above formula and dissolved in water and spread over an area of $16.8 \mathrm{~m}^{2}$ according to different treatments.

For estimation of nitrogen, phosphorus and potassium content in brinjal, a sample of $0.1 \mathrm{~g}$ each was taken from previously ground 
powder for estimation of nitrogen, $0.5 \mathrm{~g}$ for estimation of phosphorus and potassium was taken for chemical analysis. Nitrogen was estimated by Kjeldahl's method (Jackson, 1967) and expressed in percentage on dry weight basis.

Phosphorus was estimated by Vanedomolybdate method in triacid mixture as detailed by Jackson (1967). The intensity of colour developed was measured in a spectrophotometer, using blue filter. The amount of Phosphorus was calculated by referring to standard curve and expressed in percentage on dry weight basis.

Potassium content was estimated from triacid digest method using flame photometer as described by Jackson (1967).

Twenty days after transplanting, the surviving plant population in each treatment plot was counted and presented. The plant population at harvest was also counted and recorded as plants per ha. Plant height was measured in $\mathrm{cm}$ from ground level to the tip of the top most leaf at 20,40, 60 and 80 DAT for all the observational plants and averaged per plant. Number of primary branches borne on the main axis of tagged plants was counted at final harvest stage and was taken as number of primary branches. The plant spread was recorded as the product of the N-S and E-W spread of plant canopy. The marketable yield per hectare of brinjal fruits was computed and recorded accordingly in tonnes per hectare. Fisher's method of analysis of variance was applied for the analysis and interpretation of data as given by Panse and Sukhatme (1967).

\section{Results and Discussion}

Among the treatments, significantly the highest nitrogen (180.45 kg ha ${ }^{-1}$ ), phosphorus $\left(142.22 \mathrm{~kg} \mathrm{ha}^{-1}\right)$ and potassium (95.85 $\mathrm{kg} \mathrm{ha}^{-1}$ ) uptake was observed in hand weeding three times at 20, 40 and 60 DAT $\left(\mathrm{T}_{12}\right)$ and the lowest nitrogen (81.49 $\left.\mathrm{kg} \mathrm{ha}^{-1}\right)$, phosphorus $\left(73.51 \mathrm{~kg} \mathrm{ha}^{-1}\right)$ and potassium $\left(35.70 \mathrm{~kg} \mathrm{ha}^{-1}\right)$ uptake was recorded in the treatment glyphosate@1.5 kg a.i ha ${ }^{-1}$ directed spray at 25 and 50 DAT $\left(\mathrm{T}_{10}\right)\left(78.66 \mathrm{~kg} \mathrm{ha}^{-1}\right)$ which was on par with control $\left(\mathrm{T}_{13}\right)$.

Among the different integrated management practices, hand weeding three times at 20, 40 and 60 DAT $\left(\mathrm{T}_{12}\right)$ followed by intercultivation $\left(\mathrm{T}_{11}\right)$ and pendimethalin C.S as pre-emergence @ $0.70 \mathrm{~kg}$ a.i ha ${ }^{-1}+$ one hand weeding at 45 DAT $\left(\mathrm{T}_{1}\right)$ recorded significantly more nutrient uptake (N, P and $\mathrm{K}$ ) (Table 1) and it was on a par with application of oxadiargyl as preemergence @ $90 \mathrm{~g}$ a.i ha ${ }^{-1}+$ one hand weeding at 45 DAT $\left(\mathrm{T}_{2}\right)$ and oxyfluorfen preemergence @ $0.15 \mathrm{~kg}$ a.i ha ${ }^{-1}+$ one hand weeding at 45 DAT $\left(\mathrm{T}_{3}\right)$ which might be due to the better weed control efficiency, poor uptake of nutrients by weeds during grand growth period coupled with favourable conditions for growth and development of brinjal. Herbicides along with one hand weeding at 45 DAT reduced the nutrient loss from the soil by weeds when compared to herbicide alone. Similar results were recorded by Surinder Singh et al., (2005) Rameshwar et al., (2001) and Karle et al., (2010).

Among all the treatments, significantly maximum plant height was recorded in oxyfluorfen as pre-emergence@0.15 kg a.i $\mathrm{ha}^{-1}$ followed by quizalofop-ethyl @ $50 \mathrm{~g}$ a.i $\mathrm{ha}^{-1}$ at 15 to $20 \mathrm{DAT}\left(\mathrm{T}_{6}\right)(17.38 \mathrm{~cm}$, whereas the minimum plant height was recorded in control $\left(\mathrm{T}_{13}\right)(14.97 \mathrm{~cm})$.

The highest plant height was recorded in hand weeding three times at 20, 40 and 60 DAT $\left(\mathrm{T}_{12}\right)(31.85 \mathrm{~cm}$.$) , whereas the lowest plant$ height was recorded in glyphosate @ $1.5 \mathrm{~kg}$ a.i ha ${ }^{-1}$ directed spray at 25 and 50 DAT $\left(\mathrm{T}_{10}\right)$ $\left(22.55 \mathrm{~cm}\right.$.) followed by control $\left(\mathrm{T}_{13}\right)(24.32$ $\mathrm{cm})$ at 40 days after transplanting (DAT). 
Table.1 Nutrient uptake $\left(\mathrm{g}\right.$ plant $\left.{ }^{-1}\right)$ as influenced by different weed management Practices in brinjal

\begin{tabular}{|c|c|c|c|}
\hline Treatments & $\begin{array}{l}\mathrm{N} \\
(\mathrm{g} \\
\left.\text { plant }^{-1}\right)\end{array}$ & $\begin{array}{l}\mathbf{P} \\
(\mathrm{g} \\
\left.\text { plant }^{-1}\right)\end{array}$ & $\begin{array}{c}\mathbf{K} \\
(\mathrm{g} \\
\left.\text { plant }^{-1}\right)\end{array}$ \\
\hline $\begin{array}{l}\mathrm{T}_{1} \text {. Pendimethalin C.S as pre- emergence @ } 0.70 \mathrm{~kg} \mathrm{a.i} \mathrm{ha}^{-1} \\
+ \text { one hand weeding at } 45 \mathrm{DAT}\end{array}$ & 152.59 & 126.30 & 72.62 \\
\hline $\begin{array}{l}\mathrm{T}_{2-} \text { Oxadiargyl as pre-emergence @ } 90 \mathrm{~g} \text { a.i ha }{ }^{-1}+\text { one hand } \\
\text { weeding at } 45 \text { DAT }\end{array}$ & 148.68 & 115.37 & 66.24 \\
\hline $\begin{array}{l}\mathrm{T}_{3-} \text { Oxyflourfen pre-emergence @ } 0.15 \mathrm{~kg} \mathrm{a.i} \mathrm{ha}^{-1}+\text { one } \\
\text { hand weeding at } 45 \text { DAT }\end{array}$ & 136.06 & 108.77 & 64.40 \\
\hline $\begin{array}{l}\mathrm{T}_{4} \text { Pendimethalin C.S as pre-emergence @ } 0.70 \mathrm{~kg} \mathrm{a.i} \mathrm{ha}^{-1} \\
\text { followed by quizalofop- ethyl @ } 50 \mathrm{~g} \text { a.i ha }{ }^{-1} \text { at } 15 \text { to } 20 \mathrm{DAT}\end{array}$ & 125.92 & 98.28 & 60.00 \\
\hline $\begin{array}{l}\text { T5- Oxadiargyl pre-emergence @ } 90 \mathrm{~g} \text { a.i ha } \\
\text { quizalofop-ethyl } @ 50 \mathrm{~g} \text { a.i ha }{ }^{-1} \text { at } 15 \text { to } 20 \mathrm{DAT}\end{array}$ & 119.15 & 93.59 & 58.23 \\
\hline $\begin{array}{l}\mathrm{T}_{6-} \text { Oxyfluorfen pre-emergence @ } 0.15 \mathrm{~kg} \mathrm{a.i} \mathrm{ha}^{-1} \text { followed } \\
\text { by quizalofop-ethyl @ } 50 \mathrm{~g} \text { a.i ha }{ }^{-1} \text { at } 15 \text { to } 20 \mathrm{DAT}\end{array}$ & 110.54 & 88.69 & 55.22 \\
\hline $\begin{array}{l}\mathrm{T}_{7} \text { Pendimethalin C.S as pre-emergence @ } 0.70 \mathrm{~kg}^{2} . \mathrm{ha}^{-1} \\
\text { followed by propaquiza fop @ } 62.5 \mathrm{~g} \mathrm{a.i} \mathrm{ha}^{-1} \text { at } 15 \text { to } 20 \mathrm{DAT}\end{array}$ & 115.47 & 91.58 & 56.31 \\
\hline $\begin{array}{l}\mathrm{T}_{8 .} \text { Oxadiargyl pre-emergence @ } 90 \mathrm{~g} \text { a.i ha } \mathrm{ha}^{-1} \text { followed by } \\
\text { propaquiza fop @ } 62.5 \mathrm{~g} \mathrm{a.i} \mathrm{ha}{ }^{-1} \text { at } 15 \text { to } 20 \mathrm{DAT}\end{array}$ & 100.33 & 82.05 & 45.86 \\
\hline $\begin{array}{l}\mathrm{T}_{9 .} \text { Oxyfluorfen pre emergence @ } 0.15 \mathrm{~kg} \mathrm{a.i} \mathrm{ha}^{-1} \text { followed } \\
\text { by propaquiza fop @ } 62.5 \mathrm{~g} \mathrm{a.i} \mathrm{ha}^{-1} \text { at } 15 \text { to } 20 \mathrm{DAT}\end{array}$ & 112.60 & 86.45 & 48.50 \\
\hline $\begin{array}{l}\mathrm{T}_{10-} \text { Glyphosate @ } 1.5 \mathrm{~kg} \text { a.i ha }{ }^{-1} \text { directed spray } 25 \text { and } 50 \\
\text { DAT }\end{array}$ & 78.66 & 72.28 & 35.70 \\
\hline $\mathrm{T}_{11}$ - Intercultivation at 25 and $50 \mathrm{DAT}$ & 162.19 & 132.37 & 85.60 \\
\hline $\mathrm{T}_{12-}$ Hand weeding three times at 20,40 and $60 \mathrm{DAT}$ & 180.45 & 142.22 & 95.85 \\
\hline $\mathrm{T}_{13-}$ Control & 81.49 & 73.51 & 37.31 \\
\hline S.Em \pm & 3.66 & 2.19 & 1.08 \\
\hline $\mathrm{CD}(5 \%)$ & 10.75 & 6.44 & 3.18 \\
\hline
\end{tabular}


Table.2 Plant height $(\mathrm{cm})$ at different growth stages as influenced by different weed management practices in brinjal

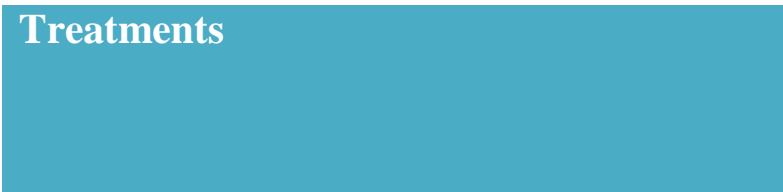

$\mathrm{T}_{1}$ - Pendimethalin C.S as pre- emergence @ $0.70 \mathrm{~kg}$ a.i $\mathrm{ha}^{-1}+$ one hand weeding at 45 DAT

T2- Oxadiargyl as pre-emergence@90 g a.i ha ${ }^{-1}+$ one hand weeding at 45 DAT

T3- Oxyflourfen pre-emergence @ 0.15 kg a.i ha ${ }^{-1}+$ one hand weeding at 45 DAT

$\mathrm{T}_{4-}$ Pendimethalin C.S as pre-emergence @ $0.70 \mathrm{~kg} \mathrm{a.i} \mathrm{ha}^{-1}$ followed by quizalofopethyl @ $50 \mathrm{~g} \mathrm{a.i} \mathrm{ha}^{-1}$ at 15 to 20 DAT

$\mathrm{T}_{5-}$ Oxadiargyl pre-emergence @ 90 g a.i ha $^{-1}$ followed by quizalofop-ethyl @ $50 \mathrm{~g}$ a.i ha ${ }^{-1}$ at 15 to 20 DAT

T6- Oxyfluorfen pre-emergence @ 0.15 kg a.i ha ${ }^{-1}$ followed by quizalofop-ethyl @ 50 $\mathrm{g}$ a.i ha $\mathrm{h}^{-1}$ at 15 to 20 DAT

$\mathrm{T}_{7 \text { - }}$ Pendimethalin C.S as pre-emergence @ $0.70 \mathrm{~kg} \mathrm{a.i} \mathrm{ha}^{-1}$ followed by propaquiza fop@ $62.5 \mathrm{~g} \mathrm{a.i} \mathrm{ha}^{-1}$ at 15 to 20 DAT

T8- Oxadiargyl pre-emergence@ 90 g a.i $\mathrm{ha}^{-1}$ followed by propaquiza fop @ $62.5 \mathrm{~g}$ a.i ha ${ }^{-1}$ at 15 to $20 \mathrm{DAT}$

T9. Oxyfluorfen pre emergence @ $0.15 \mathrm{~kg}$ a.i ha ${ }^{-1}$ followed by propaquiza fop @ 62.5 $\mathrm{g}$ a.i ha ${ }^{-1}$ at 15 to 20 DAT

$\mathrm{T}_{10 \text { - Glyphosate @ } 1.5 \text { kg a.i ha }}$ directed $^{-1}$ spray 25 and 50 DAT

$\mathrm{T}_{11}$ - Intercultivation at 25 and $50 \mathrm{DAT}$

$\mathrm{T}_{12-}$ Hand weeding three times at 20,40 and $60 \mathrm{DAT}$

\section{$\mathrm{T}_{13-}$ Control}

\section{S.Em \pm}

$\mathrm{CD}(5 \%)$

\begin{tabular}{|c|c|c|c|}
\hline $\begin{array}{c}\text { Plant } \\
\text { height at } \\
20 \text { DAT }\end{array}$ & $\begin{array}{c}\text { Plant } \\
\text { height } \\
\text { at } 40 \\
\text { DAT }\end{array}$ & $\begin{array}{c}\text { Plant } \\
\text { height } \\
\text { at } 60 \\
\text { DAT }\end{array}$ & $\begin{array}{c}\text { Plant } \\
\text { height at } \\
\text { 80 DAT }\end{array}$ \\
\hline
\end{tabular}

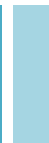

16.13

16.08

26.15

54.34

68.88

\begin{tabular}{|l|l|l|l|}
\hline 16.15 & 26.36 & 54.46 & 68.40 \\
\hline
\end{tabular}

\begin{tabular}{|l|l|l|l|}
\hline 17.16 & 29.24 & 51.3 & 65.63 \\
\hline
\end{tabular}

\begin{tabular}{|l|l|l|l|}
\hline 17.05 & 28.55 & 50.24 & 64.96 \\
\hline
\end{tabular}

\begin{tabular}{|l|l|l|l|}
\hline 17.38 & 29.71 & 50.20 & 64.49 \\
\hline
\end{tabular}

\begin{tabular}{|l|l|l|l|}
\hline 16.54 & 27.58 & 50.07 & 63.58 \\
\hline
\end{tabular}

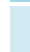

\begin{tabular}{|l|l|l|l|}
\hline 16.38 & 27.45 & 49.46 & 63.45 \\
\hline
\end{tabular}

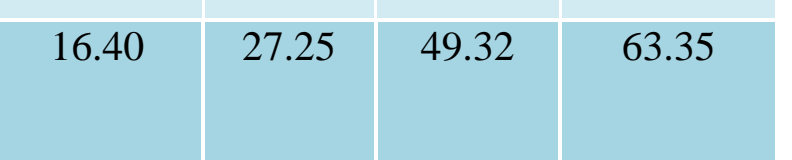

\begin{tabular}{|l|l|l|l|}
\hline 15.5 & 22.55 & 41.42 & 57.35 \\
\hline 15.72 & 31.43 & 53.25 & 71.55 \\
\hline 15.75 & 31.85 & 54.20 & 74.69 \\
\hline 14.97 & 24.32 & 44.23 & 55.46 \\
\hline 0.24 & 0.16 & 0.41 & 0.54 \\
\hline 0.98 & 0.47 & 1.22 & 1.59 \\
\hline
\end{tabular}

DAT-days after transplanting 
Table.3 Primary branches per plant, plant population and plant spread as influenced by different weed management practices in brinjal

\begin{tabular}{|c|c|c|c|c|c|}
\hline Treatments & $\begin{array}{l}\text { Primary } \\
\text { branches } \\
\text { per plant }\end{array}$ & $\begin{array}{l}\text { Initial } \\
\text { plant } \\
\text { population }\end{array}$ & $\begin{array}{l}\text { Final plant } \\
\text { population }\end{array}$ & $\begin{array}{l}\text { Plant } \\
\text { spread } \\
\left(\mathrm{m}^{-2}\right)\end{array}$ & $\begin{array}{l}\text { Fruit } \\
\text { yield } \\
\left(\mathrm{t} \mathrm{ha}^{-1}\right)\end{array}$ \\
\hline $\begin{array}{l}\text { T1 . Pendimethalin C.S as pre- emergence @ } \\
0.70 \mathrm{~kg} \text { a.i ha } \mathrm{ha}^{-1}+\text { one hand weeding at } 45 \mathrm{DAT}\end{array}$ & 6.06 & 28.57 & 28.56 & 0.31 & 27.95 \\
\hline $\begin{array}{l}\mathrm{T}_{2-} \text { Oxadiargyl as pre-emergence @ } 90 \mathrm{~g} \text { a.i } \\
\mathrm{ha}^{-1}+\text { one hand weeding at } 45 \mathrm{DAT}\end{array}$ & 5.90 & 28.57 & 28.56 & 0.33 & 27.58 \\
\hline $\begin{array}{l}\text { T3-Oxyflourfen pre-emergence @ } 0.15 \mathrm{~kg} \text { a.i } \\
\mathrm{ha}^{-1}+\text { one hand weeding at } 45 \text { DAT }\end{array}$ & 5.86 & 28.57 & 28.56 & 0.31 & 27.51 \\
\hline 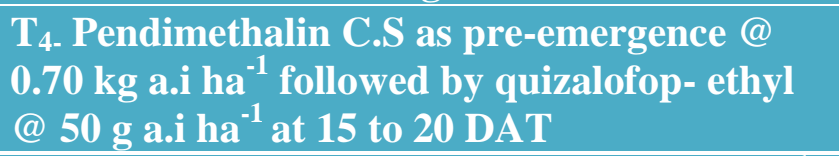 & 5.83 & 28.57 & 28.56 & 0.28 & 24.42 \\
\hline $\begin{array}{l}\mathrm{T}_{5-} \text { Oxadiargyl pre-emergence @ } 90 \mathrm{~g} \mathrm{a.i} \mathrm{ha}^{-1} \\
\text { followed by quizalofop-ethyl @ } 50 \mathrm{~g} \text { a.i ha } \\
15 \text { to } 20 \mathrm{DAT}\end{array}$ & 5.73 & 28.57 & 28.55 & 0.26 & 24.28 \\
\hline $\begin{array}{l}\text { T6-Oxyfluorfen pre-emergence @ } 0.15 \mathrm{~kg} \text { a.i } \\
\mathrm{ha}^{-1} \text { followed by quizalofop-ethyl @ } 50 \mathrm{~g} \text { a.i } \\
\mathrm{ha}^{-1} \text { at } 15 \text { to } 20 \mathrm{DAT}\end{array}$ & 5.73 & 28.57 & 28.56 & 0.26 & 24.12 \\
\hline $\begin{array}{l}\text { T. Pendimethalin C.S as pre-emergence @ } \\
0.70 \mathrm{~kg} \text { a.i ha-1 followed by propaquiza fop @ } \\
62.5 \mathrm{~g} \mathrm{a.i} \mathrm{ha}^{-1} \text { at } 15 \text { to } 20 \mathrm{DAT} \\
\end{array}$ & 5.83 & 28.57 & 28.55 & 0.27 & 23.50 \\
\hline $\begin{array}{l}\text { T8. Oxadiargyl pre-emergence @ } 90 \mathrm{~g} \mathrm{a.i} \mathrm{ha}^{-1} \\
\text { followed by propaquiza fop @ } 62.5 \mathrm{~g} \text { a.i ha } \\
\text { at } 15 \text { to } 20 \text { DAT }\end{array}$ & 5.66 & 28.57 & 28.55 & 0.28 & 23.16 \\
\hline $\begin{array}{l}\text { T9. Oxyfluorfen pre emergence @ } 0.15 \mathrm{~kg} \text { a.i } \\
\mathrm{ha}^{-1} \text { followed by propaquiza fop @ } 62.5 \mathrm{~g} \text { a.i } \\
\mathrm{ha}^{-1} \text { at } 15 \text { to } 20 \text { DAT }\end{array}$ & 5.70 & 28.57 & 28.55 & 0.28 & 23.36 \\
\hline $\begin{array}{l}\mathrm{T}_{10-} \text { Glyphosate @ } 1.5 \mathrm{~kg} \text { a.i ha }{ }^{-1} \text { directed } \\
\text { spray } 25 \text { and } 50 \text { DAT }\end{array}$ & 4.53 & 28.57 & 28.33 & 0.23 & 9.72 \\
\hline $\mathrm{T}_{11 \text { - Intercultivation at } 25 \text { and } 50 \mathrm{DAT}}$ & 6.23 & 28.57 & 28.56 & 0.32 & 28.92 \\
\hline $\begin{array}{l}T_{12} \text {. Hand weeding three times at } 20,40 \text { and } 60 \\
\text { DAT }\end{array}$ & 6.36 & 28.57 & 28.56 & 0.34 & 31.10 \\
\hline$T_{13-\text { Control }}$ & 4.50 & 28.57 & 28 & 0.20 & 10.35 \\
\hline S.Em \pm & 0.06 & - & - & 0.01 & 0.17 \\
\hline CD (5\%) & 0.18 & NS & NS & 0.03 & 0.52 \\
\hline
\end{tabular}

DAT-days after transplanting

Among all the treatments at 60 DAT, significantly the highest plant height was recorded in pendimethalin C.S as preemergence@0.70 kg a.i ha ${ }^{-1}+$ one hand weeding at 45 DAT $\left(\mathrm{T}_{1}\right)(56.52 \mathrm{~cm}$.), whereas the lowest plant height was recorded in glyphosate@1.5 kg a.i ha ${ }^{-1}$ directed spray at 25 and 50 DAT $\left(\mathrm{T}_{10}\right)(41.41 \mathrm{~cm}$.) followed by control $\left(\mathrm{T}_{13}\right)(44.32 \mathrm{~cm})$. 
At 80 days after transplanting, significantly the highest plant height was recorded in hand weeding three times at 20,40 and 60 DAT $\left(\mathrm{T}_{12}\right)(74.69 \mathrm{~cm}$.) followed by inter cultivation at 25 and 50 DAT $\left(\mathrm{T}_{11}\right)(71.55 \mathrm{~cm}$.) whereas the lowest plant height was recorded in control $\left(\mathrm{T}_{13}\right) \quad(55.46 \mathrm{~cm}$.$) followed by$ glyphosate@1.5 kg a.i ha ${ }^{-1}$ directed spray at 25 and 50 DAT $\left(\mathrm{T}_{10}\right)(57.35 \mathrm{~cm})$.

Significantly highest number of primary branches was recorded in hand weeding three times at 20, 40 and 60 DAT $\left(\mathrm{T}_{12}\right)$ (6.36), whereas the lowest number of primary branches was recorded in glyphosate @ 1.5 $\mathrm{kg}$ a.i ha ${ }^{-1}$ directed spray at 25 and 50 DAT $\left(\mathrm{T}_{10}\right) \quad$ (4.53). Other treatments were intermediate.

Different integrated weed management practices did not exhibit significant effect on Initial and final plant population. The highest plant spread was recorded in hand weeding three times at 20, 40 and 60 DAT ( $\left.\mathrm{T}_{12}\right)(0.34$ sq.m), whereas significantly the lowest plant spread was recorded in control $\left(\mathrm{T}_{13}\right)(0.20$ sq.m) which was on par with glyphosate @ $1.5 \mathrm{~kg}$ a.i ha ${ }^{-1}$ directed spray at 25 and 50 $\operatorname{DAT}\left(\mathrm{T}_{10}\right)(0.23)$.

Among the different weed management practices, oxyfluorfen pre-emergence @ 0.15 $\mathrm{kg}$ a.i ha ${ }^{-1}$ followed by quizalofop-ethyl @ 50 $\mathrm{g}$ a.i ha ${ }^{-1}$ at 15 to $20 \mathrm{DAT}\left(\mathrm{T}_{6}\right)$ recorded the highest plant height at 20 DAT (Table 2) but at 40 DAT hand weeding three times at 20, 40 and 60 DAT $\left(\mathrm{T}_{12}\right)$ was the best whereas in 60 DAT Pendimethalin C.S as pre-emergence $@ 0.70 \mathrm{~kg}$ a.i ha ${ }^{-1}+$ one hand weeding at 45 DAT $\left(\mathrm{T}_{1}\right)$ gives the highest plant height on par with oxadiargyl as pre-emergence @ $90 \mathrm{~g}$ a.i ha ${ }^{-1}+$ one hand weeding at 45 DAT $\left(\mathrm{T}_{2}\right)$, oxyfluorfen pre-emergence @ $0.15 \mathrm{~kg}$ a.i ha ${ }^{-1}$ + one hand weeding at $45 \mathrm{DAT}\left(\mathrm{T}_{3}\right)$, hand weeding three times at 20,40 and 60 DAT $\left(\mathrm{T}_{12}\right)$, and intercultivation at 25 and 50 DAT
$\left(\mathrm{T}_{11}\right)$. At $80 \mathrm{DAT}$, hand weeding three times at 20,40 and 60 DAT was the best treatment $\left(\mathrm{T}_{12}\right)$.

With regard to increase in plant height and number of primary branches per plant which could be attributed to higher availability of nutrients, particularly nitrogen and maximum utilization of sunlight by the plant due to minimum competition from weeds as a result of the actions of quizalofop (Davis, 1987) and pendimethalin (Jursik et al., 2011). This was also supported by the results of nutrient uptake studies (Table 1). Similar results were reported by Qasem (2007) Nandal and Pandita (1988).

Number of primary branches per plant, final plant population, plant spread, were highest in hand weeding three times at 20,40 and 60 DAT (T12) (Table 3), followed by inter cultivation at 25 and 50 DAT $\left(\mathrm{T}_{11}\right)$. Next best treatment was pendimethalin C.S as preemergence@0.70 kg a.i ha ${ }^{-1}+$ one hand weeding at 45 DAT $\left(\mathrm{T}_{1}\right)$ which was on par with oxadiargyl as pre-emergence @ $90 \mathrm{~g}$ a.i $\mathrm{ha}^{-1}+$ one hand weeding at $45 \mathrm{DAT}\left(\mathrm{T}_{2}\right)$ and oxyfluorfen pre-emergence @ $0.15 \mathrm{~kg}$ a.i ha ${ }^{-1}$ + one hand weeding at $45 \mathrm{DAT}\left(\mathrm{T}_{3}\right)$.

This might be due to the reason that faster vegetative growth due to reduction in weed population and the increased availability of moisture and nutrients which also enhances the early reproductive phase thereby recording minimum period for flower initiation and 50 per cent flowering and early fruit set. These results are in conformity with the findings of Sha and Karuppaiah (2005).

Among the different integrated weed management practices the treatment hand weeding three times at 20, 40 and 60 DAT $\left(\mathrm{T}_{12}\right)$ recorded the highest marketable yield per hectare (Table 3), followed by intercultivation at 25 and $50 \mathrm{DAT}\left(\mathrm{T}_{11}\right)$ and 
pendimethalin C.S as pre-emergence @ 0.70 $\mathrm{kg}$ a.i $\mathrm{ha}^{-1}+$ one hand weeding at 45 DAT $\left(\mathrm{T}_{1}\right)$ which was on par with oxadiargyl as preemergence @90 g a.i ha ${ }^{-1}+$ one hand weeding at 45 DAT $\left(\mathrm{T}_{2}\right)$ and oxyfluorfen preemergence@0.15 kg a.i ha ${ }^{-1}+$ one hand weeding at 45 DAT $\left(\mathrm{T}_{3}\right)$, which are in cuncurrence with the findings of Reddy et al., (2000).

On the basis of results obtained in this study, it was concluded that, hand weeding three times at 20, 40 and 60 DAT $\left(\mathrm{T}_{12}\right)$ followed by pendimethalin C.S as pre-emergence @0.70 $\mathrm{kg}$ a.i $\mathrm{ha}^{-1}+$ one hand weeding at 45 DAT $\left(\mathrm{T}_{1}\right)$ recorded significantly more nutrient uptake (N, P and $\mathrm{K}$ ) which has resulted in increased growth and yield in brinjal.

\section{References}

Choudhary B 1992 Vegetables, New Delhi, National Book Trust, pp. 52-59.

CMIE Centre for Monitoring Indian Economy 2010.

Davis R C 1987 Quizalofop ethyl - a new selective grass herbicide for use in broad-leaved crops. Proceedings of the Eighth Australian Weeds Conference, Sydney, New South Wales, Australia, 21-25 September, 228-230.

Jackson M L 1967 Soil Chemical Analysis. Prentice Hall, Inc., New York, USA: 498.

Jursik M, Soukup J, Holec J and Andr J 2011 Herbicide mode of actions and symptoms of plant injury by herbicides: inhibitors of cell division polymerization of microtubules inhibitors. Listy Cukrovarnicke a Reparske. 127: 2, 52-55.

Karle R L Jawale S M Dalavi N D Shaikh A A 2010 Effect of integrated weed management on yield of brinjal (Solanum melongena L.). Journal of Maharashtra Agricultural Universities. 35: 2, 321-322. 2.

Leela D 1982 A decade of weed research in horticultural crop pesticides. 15: 3-8

Nandal T R Pandita M L 1988 Studies on crop-weed competition in brinjal. Indian Journal of Weed Science. 20: 3, 9-14. 3.

Panse V G and sukhatme P V 1985 Statistical methods for agricultural workers. Indian council of agricultural research, New delhi.

Qasem J R 2007 Weed control in cauliflower with herbicides. Crop protection 26: 1013-1020.

Rameshwar Sharma G D, Vishal Dogra and Gurudev Singh 2001 Crop weed competition study in onion (Allium cepa L.) under dry temperate high hills condition of Himachal Pradesh. Indian Journal of Weed Science 33: 3/4, 168170.

Reddy C N, Reddy M D and Devi M P (2000) Efficiency of various herbicides on weed control and yield of brinjal. Indian J of Weed Sci. 32: 150-52.

Sha K Karuppaiah p 2005 integrated weed management in brinjal. Indian journal of weed science 37: 1/2 137-138.

Surinder Singh and Nandal T R 2005 Conservation of nutrients in cauliflower as influenced by weed control. Crop Research 29 (3): 453-455.

\section{How to cite this article:}

Vidyasagar K., R. V. S. K. Reddy, K. Venkata Subbaiah, M. Madhavi and Vijayapadma S. S. 2018. Weed Management Practices on Nutrient Uptake, Growth Parameters and Yield in Kharif Season Brinjal. Int.J.Curr.Microbiol.App.Sci. 7(10): 3745-3752.

doi: https://doi.org/10.20546/ijcmas.2018.710.431 